disease in WT mice within weeks, as an accelerant in lupus prone NZM2410 mice.

Methods Female and male C57BL/6J mice $(n=29)$ and pre-disease NZM2410 ( $n=31,13-15 \mathrm{wk})$ were treated with topical R848 (100 ug) or acetone vehicle $3 \mathrm{x}$ weekly for 8 weeks. 24 hour urine collection was performed to assess proteinuria by ELISA. Blood was collected to assess autoantibody levels, blood counts and renal function. Spleen and bone marrow (BM) cells were isolated for flow cytometric analysis of immune cell subsets. Spleen and renal tissue were analyzed histologically.

Results Compared to vehicle-treated mice, R848-treated B6 and NZM mice had profoundly enlarged spleens $(\mathrm{p}<0.009$ and $\mathrm{p}<0.0001$, respectively) and survival was significantly reduced $(\mathrm{p}<0.009$ and $\mathrm{p}<0.001)$. Treated B6 mice trended towards a higher ANA $(p=0.059)$, but not antidsDNA, while treated NZM mice had higher levels of ANA $(p=0.07)$ and dsDNA $(p=0.004)$. Serum IFN levels were increased in R848-treated NZM mice, correlating with earlier mortality. Albuminuria and renal pathology in treated NZM mice indicated acceleration of nephritis, but not sufficient to cause death. Consistent with this, there was no significant difference in serum BUN or creatinine between treated and untreated groups. Treated NZM mice had significantly reduced number and percent of $\mathrm{B}$ cells isolated from spleen compared with vehicle (4\% vs. $40 \%)$, as well as $\mathrm{T}$ cells $(8 \%$ vs. $31 \%)$ and pDCs $(0.10 \%$ vs. $0.75 \%)$. CD $11 b+$ cells were significantly expanded (66\% vs. $45 \%)$ in BM from treated NZM mice. IHC revealed a massive expansion of $\mathrm{F} 4 / 80+$ cells in spleens from treated NZM. Pathology of spleens in B6 and NZM mice revealed extramedullary hematopoiesis and changes consistent with histiocytic sarcoma.

Conclusions Topical TLR7/8 agonist treatment induced mild autoimmunity in B6 mice and accelerated autoimmunity in NZM2410 mice. Both had a severe immunophenotype and early death most consistent with malignant histiocytosis. Renal disease was accelerated in NZM2410 mice but was not the cause of death. Care should be taken in using TLR7/8 as a disease accelerant in NZM2410 mice as data suggest that this strain is vulnerable to death hastened by myeloprolferative disease rather than nephritis.

Funding Source(s): NIH NIAMS K08AR068471

\section{PREDICTION OF RESPONSE TO RITUXIMAB IN SLE USING A VALIDATED TWO-SCORE SYSTEM FOR INTERFERON STATUS}

${ }^{1}$ Adewonuola Alase*, 'Zoe Wigston, ${ }^{1}$ Agata Burska, ${ }^{1}$ Elizabeth Hensor, ${ }^{1}$ Antonios Psarras, ${ }^{1}$ Md Yuzaiful Md Yusof, ${ }^{2}$ John Reynolds, ${ }^{3}$ The MASTERPLANS Consortium, ${ }^{1}$ Miriam Wittmann, ${ }^{2}$ lan N Bruce, ${ }^{1}$ Edward Vital. ${ }^{1}$ University of Leeds; ${ }^{2}$ University of Manchester; ${ }^{3} \mathrm{~N} / \mathrm{A}$

\subsection{6/lupus-2019-Ism.71}

Background Rituximab is used for resistant SLE but clinical response varies. Although biomarkers of time to relapse have been validated, there are few biomarkers to predict initial response. Interferon status may predict response to rituximab and anti-TNF in RA. We previously validated two interferonstimulated gene expression scores (IFN-Score-A and IFNScore-B) that improved prediction of clinical outcomes in SLE. IFN-Score-A included most commonly reported ISGs and predicted flares and glucocorticoid requirements. IFNScore-B included ISGs that respond to multiple IFN subtypes and predicted development of SLE in At-Risk individuals. Diagnosis of SLE was associated with both scores, while only IFN-Score-B was elevated in RA. The British Society for Rheumatology Biologics Registry (BILAG-BR) collects data and samples for rituximab-treated patients in the UK. MASTERPLANS is an MRC-funded consortium to identify predictors of response.

Methods This is a preliminary analysis of the first rituximab-treated patients in the BILAG-BR with complete data. Patients were recruited if they were starting a first cycle of rituximab for active SLE (BILAG A or 2xBILAG B) despite previous cyclophosphamide or mycophenolate mofetil. Disease activity was measured using BILAG-2004. Clinical response was defined as improvement by $\geq 1$ grade in active BILAG-2004 systems with no worsening in other systems.

Whole blood was collected into TEMPUS tubes and RNA extracted. IFN-Scores were measured using a custom Taqman array as previously described, normalised to PP1A [El Sherbiny et al. Sci Rep 2018]. Multivariate logistic regression was used to test IFN-Scores and baseline clinical covariates as predictors of BILAG response at 6 months.

Results Samples were available from 147 patients, of whom 84 had complete baseline and 6 month clinical data available

Abstract 71 Table 1 predictors of response

\begin{tabular}{|c|c|c|c|c|c|c|}
\hline Predictor & Non-responders & Responders & Univariable OR $(95 \% \mathrm{Cl})$ & $\mathbf{P}$ & Multivariable OR $(95 \% \mathrm{Cl})$ & $\mathbf{P}$ \\
\hline Age (mean, 95\% Cl) & $40.5(36.2,44.8)$ & $40.9(36.2,45.6)$ & $1.015(0.993,1.037)$ & 0.188 & $0.994(0.959,1.032)$ & 0.765 \\
\hline Baseline organs affected & $22 / 44$ & $23 / 40$ & $0.866(0.468,1.601)$ & 0.645 & $1.024(0.381,2.750)$ & 0.962 \\
\hline \multicolumn{7}{|l|}{ Mucocutaneous } \\
\hline Musculoskeletal & $21 / 44$ & $18 / 40$ & $0.728(0.392,1.354)$ & 0.728 & $0.424(0.145,1.244)$ & 0.118 \\
\hline Renal & $21 / 44$ & $16 / 40$ & $0.869(0.470,1.607)$ & 0.654 & $0.290(0.087,0.969)$ & 0.044 \\
\hline Cardiovascular & $9 / 44$ & $5 / 40$ & $1.250(0.553,2.824)$ & 0.591 & $0.627(0.164,2.391)$ & 0.494 \\
\hline Neurological & $7 / 44$ & $6 / 40$ & $0.859(0.358,2.065)$ & 0.735 & $0.768(0.170,3.473)$ & 0.731 \\
\hline Antimalarial $=Y e s$ & $41 / 44$ & $39 / 40$ & $1.458(0.410,5.186)$ & 0.560 & $13.69(0.995,188.525)$ & 0.050 \\
\hline IFN-Score-A (per unit) & $2.49(1.77,3.19)$ & $1.74(1.10,2.39)$ & $0.845(0.682,1.048)$ & 0.126 & $1.601(0.935,2.743)$ & 0.086 \\
\hline IFN-Score-B (per unit) & $2.36(1.98,2.73)$ & $1.76(1.43,2.09)$ & $0.606(0.394,0.933)$ & 0.023 & $0.267(0.093,0.762)$ & 0.014 \\
\hline
\end{tabular}


Abstract 72 Table 1 LIT Responsiveness against various anchors stratified by FM status in SLE

\begin{tabular}{|c|c|c|c|c|c|c|c|c|c|}
\hline & $\Delta$ Category & $\mathrm{N}$ & Mean $\Delta$ LIT & SRM & ES & $\mathbf{N}$ & Mean $\Delta$ LIT & SRM & ES \\
\hline & ALL $(n=1356)$ & & & & & No FM & & & \\
\hline \multirow[t]{8}{*}{ T2T } & Improved & & & & & & & & \\
\hline & NO to ROFT & 13.00 & -8.80 & -0.43 & -0.39 & 11.00 & -5.5 & -0.26 & -0.29 \\
\hline & NO to RONT/LDAS & 122.00 & -3.40 & -0.18 & -0.14 & 119.00 & -3.4 & -0.18 & -0.14 \\
\hline & Same & & & & & & & & \\
\hline & No to NO & 102.00 & -0.80 & -0.05 & 0.04 & 97.00 & -0.9 & -0.05 & -0.04 \\
\hline & Worsened & & & & & & & & \\
\hline & RONT/LDAS to NO & 111.00 & 4.80 & 0.25 & 0.22 & 105.00 & 4.90 & 0.25 & 0.22 \\
\hline & ROFT to NO & 10.00 & 4.40 & 0.26 & 0.23 & 9.00 & 1.40 & 0.09 & 0.07 \\
\hline \multirow[t]{3}{*}{ SLAQ } & Improved $(\downarrow \geq 5)$ & 85.00 & -3.88 & -0.21 & -0.16 & 81.00 & -3.50 & -0.19 & -0.15 \\
\hline & Same & 904.00 & -1.12 & -0.07 & -0.05 & 853.00 & -1.00 & -0.06 & -0.05 \\
\hline & Worsened $(\uparrow \geq 5)$ & 68.00 & 3.05 & 0.14 & 0.14 & 65.00 & 2.80 & 0.13 & 0.13 \\
\hline \multirow[t]{3}{*}{ EQ5D } & Improved $(\uparrow \geq 10)$ & 309.00 & -7.60 & -0.40 & -0.33 & 292.00 & -7.50 & -0.41 & -0.34 \\
\hline & Same & 427.00 & -1.60 & -0.13 & -0.07 & 403.00 & -1.47 & -0.12 & -0.06 \\
\hline & Worsened $(\downarrow \leq 10)$ & 308.00 & 6.00 & 0.32 & 0.27 & 291.00 & 6.07 & 0.32 & 0.28 \\
\hline
\end{tabular}

and were included in this analysis. 40/84 (47.6\%) patients had BILAG response at 6 months. In univariate and multivariate analysis, high IFN-Score-B expression was significantly associated with clinical response (see table 1).

Conclusions This preliminary analysis suggests that assessment of IFN activity has a role in prediction of response to rituximab. A novel IFN score (Score B) was more predictive than classic ISGs (Score A). These results add to a body of work showing that IFN-Score-B predicts clinically significant outcomes independently of overall IFN activity. Future work will analyse this biomarker in a larger cohort of patients and integrate with other putative clinical and biological predictors of response.

Funding Source(s): Medical Research Council, National Institute of Health Research

\section{LUPUS IMPACT TRACKER IS RESPONSIVE TO CHANGES IN PHYSICIAN (T2T) AND PATIENT (SLAQ, EQ5D) RELEVANT OUTCOMES IN A LARGE SPANISH LUPUS REGISTRY COHORT}

${ }^{1}$ Iñigo Rua Figueroa, ${ }^{2}$ Herve Devilliers, ${ }^{3}$ Jose Maria Pego Reigosa, ${ }^{4}$ Irene Altabás Gonzále ${ }^{5}$ Javier Narváez-García, ${ }^{6}$ Francisco Javier López-Longo, ${ }^{6} J u a n$ Ovalles-Bonilla, ${ }^{7} J a i m e$ Calvo Alen, ${ }^{8}$ Maria Galindo Izquierdo, ${ }^{9}$ Antonio Fernandez Nebro, ${ }^{10}$ Mónica Ibáñez-Barcelo, ${ }^{11}$ Carlos Montilla Morales, ${ }^{12} \mathrm{M}^{a}$ Jesús García-Villanueva, ${ }^{13}$ Paloma Vela-Casasempere,

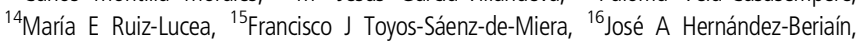
${ }^{17}$ Elvira Díez-Álvarez, ${ }^{18}$ Joel Block, ${ }^{18}$ Meenakshi Jolly. ${ }^{1}$ Hospital De Gran Canaria dr. Negrín; ${ }^{2}$ Centre Hospitalier Universitaire de Dijon; ${ }^{3}$ Complexo hospitalario Universitario Vigo; ${ }^{4}$ Hospital Do Meixoeiro; ${ }^{5}$ Hospital de Bellvitge; ${ }^{6}$ Hospital General Universitario Gregorio Marañón; ${ }^{7}$ Hospital Universitario Araba; ${ }^{8}$ Hospital Universitario 12 De Octubre; ${ }^{9}$ UGC de Reumatología, Instituto de Investigación Biomédica de Málaga (IBIMA) Hospital Regional Universitario de Málaga, Spain; ${ }^{10}$ Hospital Son Llàtzer De Palma De Mallorca; ${ }^{11}$ Hospital Clinico Universitario Salamanca; ${ }^{12}$ Hospital Ramón Y Cajal; ${ }^{13}$ Hospital General De Alicante; ${ }^{14}$ Hospital De Basurto; ${ }^{15}$ Hospital Virgen De La Macarena; ${ }^{16}$ Hospital Insular De Gran Canaria; ${ }^{17}$ Hospital De León; ${ }^{18}$ Rush University Medical Center

\subsection{6/lupus-2019-Ism.72}

Background Remission and Low Disease activity state (LDAS) are physician assessed treat to target-T2T outcomes for Systemic Lupus Erythematosus (SLE). Lupus Impact Tracker (LIT), a ten-item unidimensional patient reported tool has good psychometric properties and responds to patient reported changes in health, physician based disease activity (DA) and composite response Index (SRI). Herein we report responsiveness of LIT to changes in physician (T2T) and patient assessed outcomes (DA by SLAQ and health status (EQ5D)) among SLE patients from the largest European SLE registry- cohort.

Methods One-year longitudinal, observational, multi-center data from 1364 adult patients with SLE meeting 1997 ACR criteria were obtained from baseline and year 1 visit. This included demographics, patient reported tools (LIT, EQ5D VAS, SLAQ), SLE (activity-SLEDAI) and medications. Remission off therapy (ROFT) was defined as SLEDAI $=0$ without prednisone or Immunosuppressive/s. Remission on-therapy (RONT) was $S L E D A I=0$ and a prednisone dose $5 \mathrm{mg} /$ day and/or Immunosuppressive/s (maintenance dose). LDAS (modified) was defined as SLEDAI 4, prednisone dose $9 \mathrm{mg} /$ day and/or maintenance immunosuppressive/s. Non-optimal (NO) disease status was SLEDAI $>4$ and/or prednisone dose $>9 \mathrm{mg} /$ day and/or immunosuppressive/s in induction dose. Use of hydroxychloroquine was permitted in all groups. LIT values were compared using mixed models. Responsiveness was evaluated by standard response means (SRM) in groups with changes in DA (T2T, SLAQ) and EQ5D VAS as anchors. We did not have enough observations for stratified analysis for SLE patients with fibromyalgia.

Results 1232/1364 (90\%) were women, and 95\% were Caucasian. Mean (SD) SLEDAI and SDI were 2.6 (3.5) and 0.7 (1.1) respectively. As (i) DA was low (median 2) in LDAS, (ii) steroid use was more prevalent in RONT than LDAS, we combined RONT and LDAS into one category to analyse patient relevant differences in LIT.

LIT was responsiveness in the appropriate direction with improvement and worsening in disease activity (T2T and SLAQ) and health status (EQ5D VAS) over time. Mean LIT changes to and from NO to RONT/LDAS ranged from 3-5 (table 1), while it declined by over 8.5 with change from NO to ROFT. We had limited observations for ROFT to NO change. Mean change in LIT ranged from -3 to 3 with improvement and worsening in SLAQ, and from -7.6 to 6 with improvement and worsening in EQ5D VAS. 